\title{
Low-Density Lipoprotein Cholesterol is Inversely Associated with All-Cause Mortality of Patients in the Coronary Care Unit
}

\author{
Yingbei Weng \\ Ripeng Yin \\ Lala Qian \\ Zhi Chen \\ Jie Wang (D) \\ Huaqiang Xiang \\ Yangjing Xue \\ Kangting Ji \\ Xueqiang Guan (D)
}

Department of Cardiology, The Second Affiliated Hospital and Yuying Children's Hospital, Wenzhou Medical University, Wenzhou, 325000, Zhejiang, People's

Republic of China
Correspondence: Kangting Ji;

Xueqiang Guan

Department of Cardiology, The Second Affiliated Hospital and Yuying Children's Hospital, Wenzhou Medical University, Xueyuanxi Road, No. 109, Wenzhou, 325000, Zhejiang, People's Republic of China

Email jikt@wzmc.edu.cn; wzsgxq@163.com
Objective: The aims of this study were to investigate the relationship between low-density lipoprotein cholesterol (LDL-C) levels and all-cause mortality in coronary care unit (CCU) patients, adjusting for a wide range of potential confounding factors, to examine the potential of LDL-C in predicting the prognostic value of CCU patients.

Methods: Clinical data were extracted from Medical Information Mart for Intensive CareIII database (MIMIC-III database version v.1.4). Baseline data were collected within 24 hours after the patient was first admitted to the hospital. The primary endpoint of our study is 30-day all-cause mortality. The secondary endpoints are 90-day and one-year all-cause mortality and infections. Cox proportional hazard regression and propensity score-matched (PSM) analysis were used to analyze the association between LDL-C levels and prognostic value of CCU patients.

Results: We included a total of 1476 patients with an average age of $66.7 \pm 14.1$ years $(66 \%$ male). For 30-day all-cause mortality, the hazard ratio (95\% confidence interval) of high LDL-C level group ( $\geq 55 \mathrm{mg} / \mathrm{dl}$ ) was $0.42(0.29,0.62)$, which was compared with low LDL$\mathrm{C}$ level group $(<55 \mathrm{mg} / \mathrm{dl})$ in unadjusted model. After adjusting for age, gender and race, the association still existed $(P<0.05)$, and the HR $(95 \% \mathrm{CI})$ was $0.49(0.33,0.72)$. Further adjustment of possible covariates showed similar correlation $(P<0.05)$, and HR $(95 \% \mathrm{CI})$ was $0.65(0.43,0.97)$. Similar correlations were observed for 90 -day and one-year all-cause mortality. The relationship between all-cause mortality and LDL-C levels in CCU patients was further verified by propensity score-matched (PSM) analysis. In addition, the higher the LDL-C level, the lower the risk of infection, odds ratio (OR) values in the three models were less than $1(P<0.05)$.

Conclusion: Our data suggest that high LDL-C level is associated with a reduced risk of 30day, 90-day, and one-year mortality of patients in the CCU. And this result is still stable in the PSM model. The results need to be verified in prospective trials.

Keywords: low-density lipoprotein cholesterol, coronary care unit, all-cause mortality, cardiovascular disease

\section{Introduction}

Cardiovascular disease (CVD) has become the leading cause of death worldwide, accounting for nearly one-third of all deaths. And it accounts for $17 \%$ of the total national health expenditure in the United States. ${ }^{1,2}$ Coronary care unit (CCU) was established in the late 1960 s to reduce mortality after acute myocardial infarction by detecting and actively treating arrhythmia, and to provide clinical laboratories for the research and treatment of acute coronary syndrome. ${ }^{3,4}$ Killip et al reported that 
after the use of CCU reduced the mortality rate by nearly $20 \%$, and then CCU has been widely used and evolved into a modern CCU. ${ }^{5}$ The CCU is staffed with professionals and equipped with facilities to treat severe CVD patients. ${ }^{6,7}$ At present, we need to further improve the treatment effect of $\mathrm{CCU}$ patients, and need to find out the prognostic factors of these critically ill patients. ${ }^{8,9}$ Dyslipidemia is a heterogeneous disease composed of high LDL-C, low high density lipoprotein cholesterol (HDL-C) and high triglycerides (TG), among which LDL-C is the main sign. ${ }^{10,11}$ Evidence obtained from epidemiological studies shows that there is a linear relationship between LDL-C and the development of atherosclerotic disease. In particular, the direct link between LDL-C and coronary heart disease (CHD) risk has been demonstrated. ${ }^{12-14}$ Since LDL-C is a modifiable risk factor, all international guidelines recommend reducing plasma LDL-C concentrations in patients at risk of arteriosclerotic cardiovascular disease. ${ }^{15-17}$ The 2019 ESC/ EAS Guidelines for the management of dyslipidemia suggests that LDL-C should be controlled at $<55 \mathrm{mg} / \mathrm{dl}(1.4$ $\mathrm{mmol} / \mathrm{L}$ ) for secondary prevention in high-risk individuals and primary prevention in high-risk familial hyperlipidemia patients. ${ }^{17}$ However, it has also been shown that high LDL-C levels can improve clinical outcomes in patients with severe illness. ${ }^{16}$ How LDL-C can achieve balance in CCU patients is what we want to explore. Therefore, we used the MIMIC-III database to assess the association between outcomes in CCU patients and LDL-C.

\section{Methods}

\section{Study Population}

MIMIC-III is a large single-center database containing information about patients hospitalized in the intensive care unit of a large tertiary care hospital. MIMIC-III v.1.4 contains data on 53,423 admissions to different hospitals of adult patients (16 years of age or older) admitted to the intensive care unit between 2001 and 2012. The database includes basic patient information, treatment details and survival data. ${ }^{18}$ MIMIC-III has obtained approval from the Institutional Review Boards of the Beth Israel Deaconess Medical Center and the Massachusetts Institute of Technology. Our institutional ethics review committee does not need to approve research protocols and informed consent, because our research involves retrospective analysis of public databases.
Among the more than 50,000 patients in the database, the included subjects met the following criteria: (1) CCU patients; (2) length of hospital stay $>2$ days; (3) age $\geq 16$ years. The exclusion criteria are as follows: (1) the patient's individual data is missing by more than $10 \%$; (2) outliers: the value exceeds the mean \pm 3 Standard Deviation (SD).

\section{Covariates}

The data on the first day of CCU admission were extracted from MIMIC III using Structured Query Language (SQL) including demographic data, basic vital signs, comorbidities, basic laboratory parameters and scoring system before treatment. Demographic information included age, gender, race. Complications included coronary heart disease, congestive heart failure (CHF), cardiogenic shock (CS), arrhythmia, heart valve disease, peripheral vascular disease, hypertension and liver disease. Liver disease refers to the pathological changes that occur in the liver, including viral hepatitis, cirrhosis, fatty liver, alcoholic liver disease, portal hypertension, hepatic encephalopathy, hepatorenal syndrome, liver necrosis, and many other liver diseases and their complications. Excluding liver cancer. Laboratory parameters included LDL-C, white blood cell (WBC) count, urea nitrogen, serum creatinine, total cholesterol (TC), TG, HDL-C and non-high density lipoprotein cholesterol (non-HDL-C) in the first 24 hours. In addition, simplified acute physiology score II (SAPSII) and acute physiology score III (APSIII) were also included. These two scores are applicable to critically ill patients to evaluate the severity of the patient's condition.

\section{Outcomes}

The primary endpoint of our study is 30-day all-cause mortality. The secondary endpoints are 90-day and one-year allcause mortality and infections. The observation time was from the patient's first admission to the hospital until death. The date of death was collected by MIMIC-III database from the records in the Social Security Death Index.

\section{Statistical Analyses}

According to the LDL-C value, the study participants were divided into two groups $(<55 \mathrm{mg} / \mathrm{dl}$ and $\geq 55 \mathrm{mg} / \mathrm{dl}$ ). Categorical variables were expressed by frequency (percentage) and compared with Chi-square test or Fisher's exact test. Continuous variables were represented by the mean (SD), and Kruskal-Wallis $H$-test or variance analysis was used to compare the different groups.

Cox proportional hazard regression was used to evaluate the association between all-cause mortality and LDL-C, and 


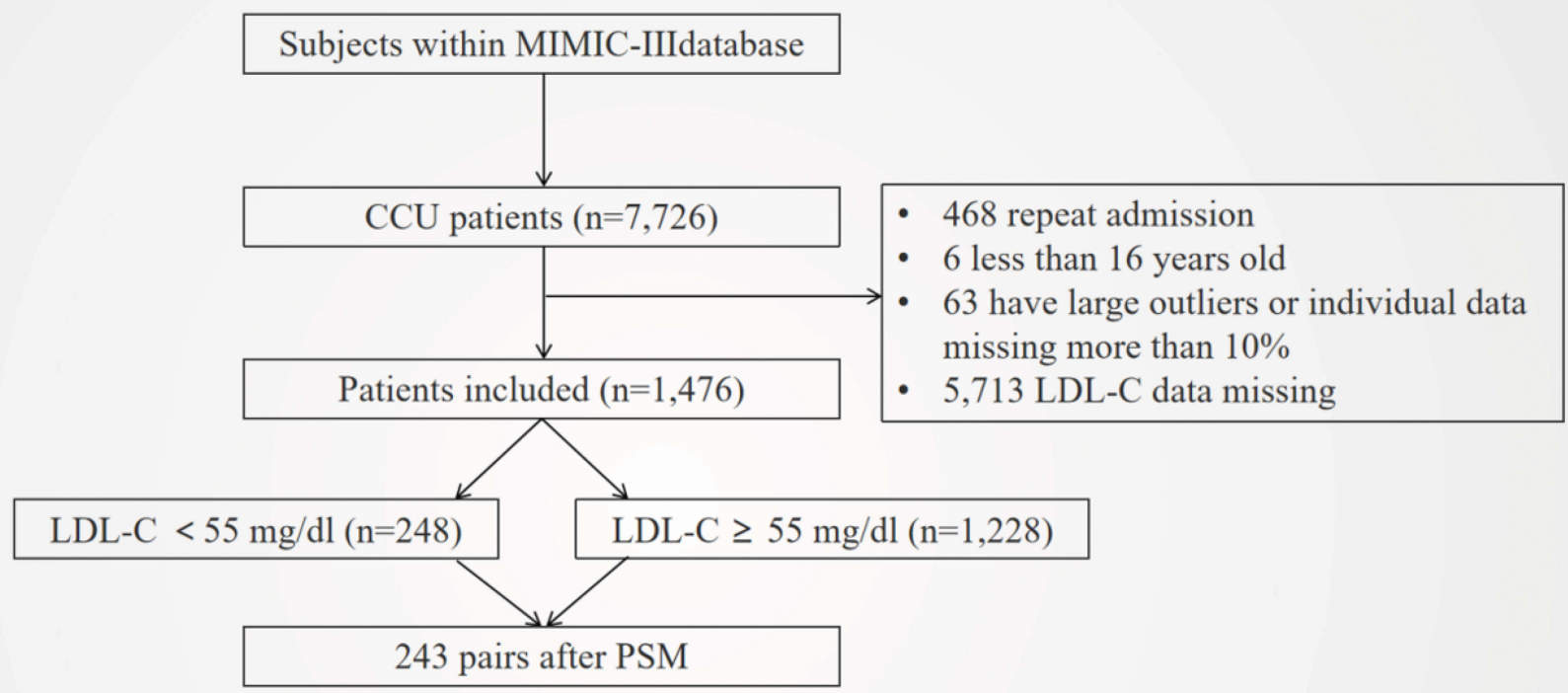

Figure I Flowchart of included patients.

Abbreviation: MIMIC III, Multiparameter Intelligent Monitoring in Intensive Case Database III; CCU, coronary care unit; LDL-C, low-density lipoprotein cholesterol; PSM, propensity-score matching.

the result was shown as a hazard ratio (HR) with a 95\% confidence interval (CI). ${ }^{19}$ Three multivariate analysis models were established for each end point, and the low LDL-C level group $(<55 \mathrm{mg} / \mathrm{dl}$ ) was set as the reference group. The choice of covariates was based on the estimated value of impact $>$ $10 \%{ }^{20}$ In model 1 , the covariates were not adjusted; in model 2 , the covariates included age, gender and race; in model 3, age, gender, race, serum urea nitrogen, serum creatinine, mean arterial pressure, coronary heart disease, cardiogenic shock were further adjusted. To avoid potential bias, and because of differences in baseline characteristics, the propensity score matching (PSM) was used. ${ }^{21}$ PSM was performed at a ratio of $1: 1$ using a caliper width of 0.02 of the SD of the logit of the propensity score. Then PSM model was analyzed. Subgroup analysis was performed to explore if the association differed for subgroups classified using different parameters including age, sex, race, CHD, CS, CHF, arrhythmia, heart valve disease, peripheral vascular disease and hypertension.

All analyses were conducted using $\mathrm{R}$ software (Version 3.6.1, http://www.r-project.org). All of the tests were twosided and $P<0.05$ indicated a significant difference.

\section{Results}

\section{Characteristics of Patients}

We identified 1476 patients who met our inclusion criteria (Figure 1). The eligible participants included 509 women and 982 men with a mean age of $66.7 \pm 14.1$ years, and a mean LDL-C of 89.79. Baseline characteristics are shown in Table 1. According to the LDL-C value, the study participants were divided into two groups $(<$ $55 \mathrm{mg} / \mathrm{dl}$ and $\geq 55 \mathrm{mg} / \mathrm{dl}$ ) may have protective functions.

They suffered more from CHD. However, patients with lower LDL-C levels were more likely to suffer from $\mathrm{CHF}$, $\mathrm{CS}$, and arrhythmia. Patients with higher LDL-C also had higher levels of other blood lipids, including TC, TG, HDL-C, and non-HDL-C. The serum creatinine and urea nitrogen levels were significantly lower in patients with higher LDL-C. And the SAPSII score and APSIII score were lower. (All $P<0.05$ ).

\section{Association Between LDL-C and Mortality}

We first conducted analysis of the relationship between lipids and the prognosis of CCU patients, including TC, TG, nonHDL-C and remnant lipoprotein cholesterol (RLP-C). The results show that higher blood lipid levels are related to better prognosis of CCU severely ill patients (Table S1). After that, we conducted a detailed analysis of LDL-C. After adjusting for possible covariates, different models were established to evaluate the relationship between LDL-C and the prognosis of CCU patients. The results of these relationships are shown in Table 2. For 30-day all-cause mortality, HR (95\% CI) of high LDL-C level group ( $\geq 55 \mathrm{mg} / \mathrm{dl}$ ) was $0.42(0.29,0.62)$ in unadjusted model compared with low LDL-C level group 
Table I Baseline Characteristics of the Study Population

\begin{tabular}{|c|c|c|c|}
\hline \multirow[t]{2}{*}{ Characteristics } & \multicolumn{2}{|c|}{ LDL-C Level, mg/dl } & \multirow[t]{2}{*}{$P$ value } \\
\hline & $<55(n=248)$ & $\geq 55(n=1228)$ & \\
\hline \multicolumn{4}{|l|}{ Clinical parameters, $n(\%)$} \\
\hline Age, years & $70.69 \pm 13.68$ & $65.87 \pm 14.05$ & $<0.001$ \\
\hline Sex, n (\%) & & & 0.808 \\
\hline Male & $162(65.32)$ & $812(66.12)$ & \\
\hline Female & $86(34.68)$ & $416(33.88)$ & \\
\hline Ethnicity, n(\%) & & & 0.113 \\
\hline White & $177(7 \mid .37)$ & $792(64.50)$ & \\
\hline Black & $9(3.63)$ & $59(4.80)$ & \\
\hline Other & $62(25.00)$ & $377(30.70)$ & \\
\hline SBP, mmHg & $113.92 \pm 16.45$ & $116.43 \pm 15.54$ & 0.022 \\
\hline $\mathrm{DBP}, \mathrm{mmHg}$ & $58.84 \pm 10.95$ & $63.01 \pm 10.67$ & $<0.001$ \\
\hline MAP, mmHg & $76.48 \pm 11.42$ & $80.08 \pm 10.48$ & $<0.001$ \\
\hline Heart rate, beats/minute & $78.30 \pm 15.98$ & $77.46 \pm 13.99$ & 0.405 \\
\hline Respiratory rate, times/minute & $|8.73 \pm 3.4|$ & $18.40 \pm 3.10$ & 0.132 \\
\hline Temperature, ${ }^{\circ} \mathrm{C}$ & $36.71 \pm 0.68$ & $36.76 \pm 0.53$ & 0.204 \\
\hline \multicolumn{4}{|l|}{ Comorbidities } \\
\hline Coronary heart disease, $n(\%)$ & $149(60.08)$ & $867(70.60)$ & 0.001 \\
\hline Congestive heart failure, $n(\%)$ & $117(47.18)$ & $507(41.35)$ & 0.091 \\
\hline Cardiogenic shock, n(\%) & $38(15.32)$ & III (9.04) & 0.003 \\
\hline Arrhythmia, n (\%) & $127(5 \mid .21)$ & 505 (41.19) & 0.004 \\
\hline Heart valve disease, $\mathrm{n}(\%)$ & $46(18.55)$ & $202(16.48)$ & 0.426 \\
\hline Peripheral vascular disease, $\mathrm{n}(\%)$ & $25(10.08)$ & $93(7.59)$ & 0.187 \\
\hline Hypertension, n (\%) & I58 (63.7I) & $739(60.18)$ & 0.299 \\
\hline Liver disease, n (\%) & $14(5.65)$ & $44(3.59)$ & 0.129 \\
\hline \multicolumn{4}{|l|}{ Laboratory parameters } \\
\hline White blood cell count, $10^{9} / \mathrm{L}$ & $11.34 \pm 4.82$ & $1 \mathrm{I} .46 \pm 4.38$ & 0.692 \\
\hline Serum creatinine, mg/dl & $1.57 \pm 1.41$ & $1.20 \pm 0.94$ & $<0.001$ \\
\hline Serum urea nitrogen, $\mathrm{mg} / \mathrm{dl}$ & $29.48 \pm 21.30$ & $22.34 \pm 13.81$ & $<0.001$ \\
\hline Total cholesterol, mg/dl & $109.76 \pm 33.65$ & $|7| .05 \pm 37.60$ & $<0.001$ \\
\hline Triglycerides, mg/dl & $134.59 \pm|34.6|$ & $135.69 \pm 103.60$ & 0.026 \\
\hline $\mathrm{HDL}-\mathrm{C}, \mathrm{mg} / \mathrm{dl}$ & $41.22 \pm 15.28$ & $45.72 \pm 14.22$ & $<0.001$ \\
\hline Non-HDL-C, mg/dl & $68.54 \pm 30.44$ & $125.45 \pm 35.56$ & $<0.001$ \\
\hline \multicolumn{4}{|l|}{ Scoring systems } \\
\hline SAPSII & $36.22 \pm 14.33$ & $29.64 \pm 11.29$ & $<0.001$ \\
\hline APSIII & $36.32 \pm \mid 4.41$ & $29.60 \pm 11.28$ & $<0.001$ \\
\hline \multicolumn{4}{|l|}{ Mortality } \\
\hline 30-day & $38(15.32)$ & $82(6.68)$ & $<0.001$ \\
\hline 90-day & $5 \mathrm{I}(20.56)$ & 107 (8.7I) & $<0.001$ \\
\hline One year & $73(29.44)$ & $177(14.4 I)$ & $<0.001$ \\
\hline
\end{tabular}

Notes: Liver disease refers to the pathological changes that occur in the liver, including viral hepatitis, cirrhosis, fatty liver, alcoholic liver disease, portal hypertension, hepatic encephalopathy, hepatorenal syndrome, liver necrosis, and many other liver diseases and their complications. Excluding liver cancer.

Abbreviations: LDL-C, low-density lipoprotein cholesterol; SBP, systolic blood pressure; DBP, diastolic blood pressure; MAP, mean arterial pressure; HDL-C, high density lipoprotein cholesterol; Non-HDL-C, non-high density lipoprotein cholesterol; SAPSII, simplified acute physiology score II; APSIII, acute physiology score III. 


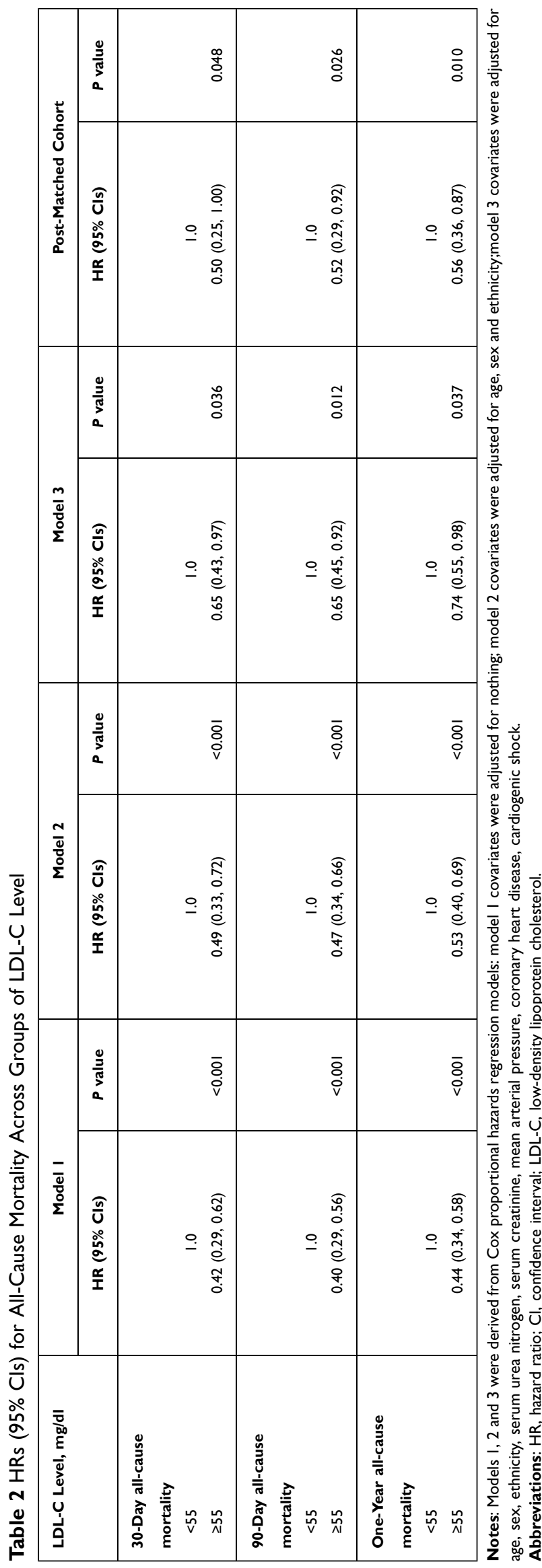

$(<55 \mathrm{mg} / \mathrm{dl})$. After adjusting for age, gender and race, the association still existed $(P<0.05)$, and the HR $(95 \% \mathrm{CI})$ was $0.49(0.33,0.72)$. Further adjustment of possible covariates showed similar correlation in model $3(\mathrm{P}<0.05)$, HR $(95 \%$ $\mathrm{CI}$ ) was $0.65(0.43,0.97)$. A similar correlation was observed between 90 -day and one-year all-cause mortality. In addition, LDL-C values were divided into four groups and five groups respectively, and different models were established to evaluate the relationship between LDL-C and prognosis of CCU patients (Table S2). For 30-day all-cause mortality, the HR ( $95 \% \mathrm{CI}$ ) of the group with the highest LDL-C level was less than 1 in all three models $(\mathrm{P}<0.05)$, regardless of whether the LDL-C was divided into four or five groups. A similar correlation was observed between 90-day and one-year allcause mortality.

\section{PSM Analysis}

PSM analysis was conducted to assess the relationship between LDL-C and the all-cause mortality of CCU patients. The baseline characteristics of patients in different LDL-C groups did not significantly differ (Table 3 ). Cox proportional hazard regression analysis revealed that high LDL-C level was independently related to better prognosis of CCU patients (Table 2). For 30-day, 90-day and one-year all-cause mortality, HR $(95 \% \mathrm{CI})$ were 0.50 $(0.25,1.00), 0.52(0.29,0.92)$ and $0.56(0.36,0.87)$.

\section{Association Between LDL-C and Infection}

We analyzed the relationship between LDL-C and the risk of infection in CCU patients (Table 4). OR (95\% CI) of high LDL-C level group ( $\geq 55 \mathrm{mg} / \mathrm{dl})$ was $0.43(0.31$, $0.60), 0.48(0.35,0.68), 0.63(0.44,0.92)$ in three models compared with low LDL-C level group $(<55 \mathrm{mg} / \mathrm{dl})$ respectively (All $P<0.05$ ).

\section{Subgroup Analyses}

Subgroup analysis results are shown in Table 5. In the subgroup analysis, we analyzed the common diseases of CCU patients, and no significant interaction was observed. It shows that the results of this article were stable in $\mathrm{CCU}$ patients.

\section{Discussion}

Cardiovascular disease is a heavy burden on the medical system, especially because of its increasing mortality and incidence rate. ${ }^{22,23}$ Over the past 50 years, increased levels 
Table 3 Characteristics of the Study Population After PSM

\begin{tabular}{|c|c|c|}
\hline \multirow[t]{2}{*}{ Characteristics } & \multicolumn{2}{|c|}{ LDL-C Level, mg/dl } \\
\hline & $<55(n=243)$ & $\geq 55(n=243)$ \\
\hline Clinical parameters, $n(\%)$ & & \\
\hline Age, years & $70.81 \pm 13.58$ & $64.13 \pm 13.40$ \\
\hline \multicolumn{3}{|l|}{ Sex, n (\%) } \\
\hline Male & $159(65.4)$ & $160(65.8)$ \\
\hline Female & $84(34.6)$ & $83(34.2)$ \\
\hline \multicolumn{3}{|l|}{ Ethnicity, n(\%) } \\
\hline White & $175(72)$ & $157(64.6)$ \\
\hline Black & $8(3.3)$ & $18(7.4)$ \\
\hline Other & $60(24.7)$ & $68(28)$ \\
\hline MAP, $\mathrm{mmHg}$ & $76.53 \pm 11.48$ & $80.99 \pm 11.16$ \\
\hline Heart rate, beats/minute & $78.49 \pm 15.88$ & $78.85 \pm 15.17$ \\
\hline Respiratory rate, times/minute & $|8.7| \pm 3.4 \mid$ & $19.06 \pm 3.29$ \\
\hline \multicolumn{3}{|l|}{ Comorbidities } \\
\hline Coronary heart disease, $\mathrm{n}(\%)$ & $146(59.8)$ & $179(73.4)$ \\
\hline Congestive heart failure, $n(\%)$ & $115(47.1)$ & $13 \mid(53.7)$ \\
\hline Cardiogenic shock, n(\%) & $36(14.8)$ & $46(18.9)$ \\
\hline Arrhythmia, n (\%) & $126(51.6)$ & $99(40.6)$ \\
\hline Heart valve disease, n (\%) & $45(18.4)$ & $46(18.9)$ \\
\hline Peripheral vascular disease, $\mathrm{n}(\%)$ & $25(10.2)$ & $34(13.9)$ \\
\hline Hypertension, n (\%) & $157(64.3)$ & $164(67.2)$ \\
\hline Liver disease, n (\%) & $14(5.8)$ & $9(3.7)$ \\
\hline \multicolumn{3}{|l|}{ Laboratory parameters } \\
\hline White blood cell count, $10^{9} / \mathrm{L}$ & $11.29 \pm 4.80$ & $12.17 \pm 4.50$ \\
\hline Serum creatinine, $\mathrm{mg} / \mathrm{dl}$ & $1.57 \pm 1.42$ & $1.31 \pm 1.04$ \\
\hline Serum urea nitrogen, $\mathrm{mg} / \mathrm{dl}$ & $29.78 \pm 21.41$ & $24.35 \pm 15.13$ \\
\hline \multicolumn{3}{|l|}{ Mortality } \\
\hline 30-day & $38(15.6)$ & $16(6.6)$ \\
\hline 90-day & $50(20.6)$ & $21(8.6)$ \\
\hline One year & $72(29.6)$ & $36(14.8)$ \\
\hline
\end{tabular}

Notes: I:I matching for age, sex, ethnicity, mean arterial pressure, heart rate, respiratory rate, coronary heart disease, congestive heart failure, cardiogenic shock, arrhythmia, heart valve disease, peripheral vascular disease, hypertension, liver disease, white blood cell, serum urea nitrogen, and creatinine.

Abbreviations: PSM, propensity-score matching; LDL-C, low-density lipoprotein cholesterol; MAP, mean arterial pressure.

of CCU have been associated with improved outcomes in critically ill patients. ${ }^{24}$ Therefore, it is very important to study the early useful biomarkers of CCU prognosis. Few studies have discussed the relationship between LDL-C and mortality in CCU patients. As an easily available biomarker, LDL$\mathrm{C}$ has certain value for the prognosis of CCU patients and can play a role in rapid clinical evaluation.

The exact mechanism of the relationship between LDL-C and mortality in CCU patients remains unclear.
LDL-C is a large molecule comprised of many proteins as well as lipids including cholesterol, phospholipids, and triglycerides. As we all known that the circulating levels of LDL are directly associated with atherosclerosis disease severity. $^{25}$ However, lipoproteins, including LDL-C, in addition to playing a role in lipid transport, may have protective functions. $^{26}$ Systemic inflammatory response syndrome (SIRS) refers to systemic inflammatory response, that is, the body's response to a variety of cytokines/inflammatory mediators. Both infectious and non-infectious factors can activate inflammatory cells through different ways, release pro-inflammatory mediators such as TNF- $\alpha$, IL-1, and participate in the body's defense response to resist external damage and stimulation. These inflammatory cells further promote the activation of inflammatory cells and form a "cascade effect" in the body, leading to a continuous increase in the number of inflammatory mediators and an expanding inflammatory response. When the body's compensatory ability is exceeded, the body has an excessive inflammatory response, causing extensive tissue cell damage, resulting in systemic inflammatory cell response syndrome. ${ }^{27,28}$ Cardiovascular critically ill patients suffer fatal cardiovascular trauma, and a series of inflammatory cascades are produced in the body. The host response during this period is mediated by cytokines and growth factors, which are capable of influencing lipid metabolism. ${ }^{29,30}$ Endogenous LDL can protect against the lethal effects of inflammatory. At least part of this protection is achieved through decreased in vivo production of pro-inflammatory cytokines, in spite of increased cytokine production capacity. $^{31}$ And the results of this article show that a higher level of LDL-C is associated with a lower risk of infection, this may explain our results.

Our study had several strengths. The sample size in this study was large enough to identify a significant association between LDL-C and all-cause mortality of CCU patients. Our analysis adjusted for potential confounding factors that might influence the relationship between LDL-C and all-cause mortality of CCU patients, and we have repeatedly verified our results with multiple models. However, our research has some limitations. Firstly, we adopted a single-center retrospective study design. Therefore, there may be some selection biases in our study. In the future, forward-looking multi-center research is needed to solve this problem. Secondly, the baseline levels of LDL-C could be affected by the use of lipid-lowering drugs before CCU admission. We extracted data from the first 
Table 4 ORs (95\% Cls) for Infection Across Groups of LDL-C Level

\begin{tabular}{|c|c|c|c|c|c|c|}
\hline \multirow[t]{2}{*}{ LDL-C Level, mg/dl } & \multicolumn{2}{|c|}{ Model I } & \multicolumn{2}{|c|}{ Model 2} & \multicolumn{2}{|c|}{ Model 3} \\
\hline & OR (95\% Cls) & $P$ value & OR (95\% Cls) & $P$ value & OR (95\% Cls) & $P$ value \\
\hline \multicolumn{7}{|l|}{ Infection } \\
\hline$<55$ & 1.0 & & 1.0 & & 1.0 & \\
\hline$\geq 55$ & $0.43(0.3 \mathrm{I}, 0.60)$ & $<0.001$ & $0.48(0.35,0.68)$ & $<0.001$ & $0.63(0.44,0.92)$ & 0.015 \\
\hline
\end{tabular}

Notes: Models I, 2 and 3 were derived from logistic regression models: model I covariates were adjusted for nothing; model 2 covariates were adjusted for age, sex and ethnicity;model 3 covariates were adjusted for age, sex, ethnicity, serum urea nitrogen, serum creatinine, mean arterial pressure, coronary heart disease, cardiogenic shock. Abbreviation: OR, odds ratio.

Table 5 Subgroup Analysis of the Associations Between 30-Day All-Cause Mortality and the LDL-C Level

\begin{tabular}{|c|c|c|c|c|}
\hline & \multirow[t]{2}{*}{ No. of Patients } & \multicolumn{2}{|c|}{ LDL-C Level, mg/dl } & \multirow[t]{2}{*}{$P$ for Interaction } \\
\hline & & $<55$ & $\geq \mathbf{5 5}$ & \\
\hline Age, years & & & & 0.052 \\
\hline$<70$ & 737 & 1.0 & $1.77(0.50,6.30)$ & \\
\hline$\geq 70$ & 739 & 1.0 & $0.53(0.34,0.82)$ & \\
\hline Sex & & & & 0.258 \\
\hline Male & 974 & 1.0 & $0.49(0.29,0.83)$ & \\
\hline Female & 502 & 1.0 & $0.79(0.4 \mathrm{I}, \mathrm{I} .52)$ & \\
\hline Ethnicity & & & & 0.200 \\
\hline White & 969 & 1.0 & $0.54(0.33,0.91)$ & \\
\hline Black & 68 & 1.0 & $0.70(0.34, \mathrm{I} .42)$ & \\
\hline Other & 439 & 1.0 & $0.20(0.30,1.39)$ & \\
\hline Coronary heart disease & & & & 0.100 \\
\hline Yes & 1016 & 1.0 & $0.83(0.50,1.38)$ & \\
\hline No & 460 & 1.0 & $0.41(0.20,0.82)$ & \\
\hline Cardiogenic shock & & & & 0.302 \\
\hline Yes & 149 & 1.0 & $0.86(0.43,1.74)$ & \\
\hline No & 1327 & 1.0 & $0.55(0.33,0.92)$ & \\
\hline Congestive heart failure & & & & 0.195 \\
\hline Yes & 624 & 1.0 & $0.52(0.3 \mathrm{I}, 0.87)$ & \\
\hline No & 850 & 1.0 & $0.96(0.46,1.99)$ & \\
\hline Arrhythmia & & & & 0.972 \\
\hline Yes & 632 & 1.0 & $0.67(0.40,1.12)$ & \\
\hline No & 842 & 1.0 & $0.66(0.32,1.36)$ & \\
\hline Peripheral vascular disease & & & & 0.356 \\
\hline Yes & 118 & 1.0 & $0.63(0.4 \mathrm{I}, 0.97)$ & \\
\hline No & 1356 & 1.0 & $\mathrm{I} .28(0.30,5.4 \mathrm{I})$ & \\
\hline Hypertension & & & & 0.707 \\
\hline Yes & 897 & 1.0 & $0.70(0.36, \mathrm{I} .36)$ & \\
\hline No & 579 & 1.0 & $0.60(0.36,1.02)$ & \\
\hline Heart Valve disease & & & & 0.918 \\
\hline Yes & 248 & 1.0 & $0.66(0.4 \mathrm{I}, \mathrm{I} .05)$ & \\
\hline No & 1226 & 1.0 & $0.69(0.28,1.68)$ & \\
\hline
\end{tabular}

Notes: HRs (95\% Cls) were derived from Cox proportional hazards regression models. Covariates were adjusted as in model 3(Table 2). 
measurement when the patient was admitted to the hospital. Perhaps measuring the difference in LDL-C before and after admission may be more helpful than a single test. Thirdly, LDL-C is easy to obtain in clinical practice, but the loss of LDL-C in the database is still common, which may lead to selection bias.

\section{Conclusions}

We proved that high levels of LDL-C are associated with better short-term and long-term prognosis in CCU patients. This should be confirmed in prospective studies.

\section{Funding}

This work was not supported by funding.

\section{Disclosure}

The authors declare that there is no conflict of interest.

\section{References}

1. Stram DO, Liu Y, Henderson KD, et al. Age-specific effects of hormone therapy use on overall mortality and ischemic heart disease mortality among women in the California Teachers Study. Menopause. 2011;18(3):253-261. doi:10.1097/gme.0b013e 3181f0839a

2. Mahmood SS, Levy D, Vasan RS, et al. The Framingham Heart Study and the epidemiology of cardiovascular disease: a historical perspective. Lancet. 2014;383(9921):999-1008. doi:10.1016/S01406736(13)61752-3

3. Lee TH, Goldman L. The coronary care unit turns 25: historical trends and future directions. Ann Intern Med. 1988;108(6):887-894. doi:10.7326/0003-4819-108-6-887

4. Todo MC, Bergamasco CM, Azevedo PS, et al. Impact of coronary intensive care unit in treatment of myocardial infarction. Rev Assoc Med Bras. 2017;63(3):242-247. doi:10.1590/1806-9282.63.03.242

5. Killip T 3rd, Kimball JT. Treatment of myocardial infarction in a coronary care unit. A two year experience with 250 patients. $\mathrm{Am}$ J Cardiol. 1967;20(4):457-464. doi:10.1016/0002-9149(67)90023-9

6. Katz JN, Minder M, Olenchock B, et al. The genesis, maturation, and future of critical care cardiology. J Am Coll Cardiol. 2016;68 (1):67-79. doi:10.1016/j.jacc.2016.04.036

7. Kasaoka S. Evolved role of the cardiovascular intensive care unit (CICU). J Intensive Care. 2017;5(1):72. doi:10.1186/s40560-0170271-7

8. Teskey RJ, Calvin JE, McPhail I. Disease severity in the coronary care unit. Chest. 1991;100(6):1637-1642. doi:10.1378/ chest.100.6.1637

9. Fye WB. Resuscitating a circulation abstract to celebrate the 50th anniversary of the coronary care unit concept. Circulation. 2011;124 (17):1886-1893. doi:10.1161/CIRCULATIONAHA.111.033597

10. Alasnag M, Awan Z, Al Ghamdi A, Al Modaimeigh H, Al Shemiri M. Improvement initiative in LDL-C management in Saudi Arabia: a call to action. Int J Cardiol Heart Vasc. 2020;31:100667.

11. Klop B, Elte JW, Cabezas MC. Dyslipidemia in obesity: mechanisms and potential targets. Nutrients. 2013;5(4):1218-1240. doi:10.3390/ nu5041218

12. Maron DJ, Fazio S, Linton MF. Current perspectives on statins. Circulation. 2000;101(2):207-213. doi:10.1161/01.CIR.101.2.207
13. Ference BA, Ginsberg HN, Graham I, et al. Low-density lipoproteins cause atherosclerotic cardiovascular disease. 1. Evidence from genetic, epidemiologic, and clinical studies. A consensus statement from the European atherosclerosis society consensus panel. Eur Heart J. 2017;38(32):2459-2472. doi:10.1093/eurheartj/ehx144

14. Lloyd-Jones DM, Morris PB, Ballantyne CM, et al. 2017 focused update of the 2016 ACC expert consensus decision pathway on the role of non-statin therapies for LDL-cholesterol lowering in the management of atherosclerotic cardiovascular disease risk: a report of the American college of cardiology task force on expert consensus decision pathways. J Am Coll Cardiol. 2017;70(14):1785-1822.

15. Piepoli MF, Hoes AW, Agewall S, et al. 2016 European guidelines on cardiovascular disease prevention in clinical practice: the sixth joint task force of the European society of cardiology and other societies on cardiovascular disease prevention in clinical practice (constituted by representatives of 10 societies and by invited experts)developed with the special contribution of the European Association for Cardiovascular Prevention \& Rehabilitation (EACPR). Eur Heart J. 2016;37(29):2315-2381.

16. Grundy SM, Stone NJ, Bailey AL, et al. 2018 AHA/ACC/AACVPR/ AAPA/ABC/ACPM/ADA/AGS/APhA/ASPC/NLA/PCNA guideline on the management of blood cholesterol: a report of the American college of cardiology/American heart association task force on clinical practice guidelines. Circulation. 2019;139(25):e1082-e1143.

17. Mach F, Baigent C, Catapano AL, et al. 2019 ESC/EAS guidelines for the management of dyslipidaemias: lipid modification to reduce cardiovascular risk. Eur Heart J. 2020;41(1):111-188.

18. Johnson AEW, Pollard TJ, Shen L, et al. MIMIC-III, a freely accessible critical care database. Sci Data. 2016;3(1):160035. doi:10.1038/ sdata.2016.35

19. Sun H, Que J, Peng Y, et al. The neutrophil-lymphocyte ratio: a promising predictor of mortality in coronary care unit patients a cohort study. Int Immunopharmacol. 2019;74:105692. doi:10.1016/ j.intimp.2019.105692

20. Jaddoe VW, de Jonge LL, Hofman A, et al. First trimester fetal growth restriction and cardiovascular risk factors in school age children: population based cohort study. BMJ. 2014;348(jan23 1):g14. doi:10.1136/bmj.g14

21. Zhu L, She Z-G, Cheng X, et al. Association of blood glucose control and outcomes in patients with COVID-19 and pre-existing type 2 diabetes. Cell Metab. 2020;31(6):1068-1077.e3. doi:10.1016/j. cmet.2020.04.021

22. Katzmarzyk PT, Church TS, Craig CL, et al. Sitting time and mortality from all causes, cardiovascular disease, and cancer. Med Sci Sports Exerc. 2009;41(5):998-1005. doi:10.1249/MSS.0b013e3181930355

23. Garcia M, Mulvagh SL, Bairey Merz CN, et al. Cardiovascular disease in women: clinical perspectives. Circ Res. 2016;118 (8):1273-1293. doi:10.1161/CIRCRESAHA.116.307547

24. Peng Y, Guan X, Wang J, et al. Red cell distribution width is correlated with all-cause mortality of patients in the coronary care unit. J Int Med Res. 2020;48(7):300060520941317. doi:10.1177/ 0300060520941317

25. Rhoads JP, Major AS. How oxidized low-density lipoprotein activates inflammatory responses. Crit Rev Immunol. 2018;38 (4):333-342. doi:10.1615/CritRevImmunol.2018026483

26. Feingold KR, Funk JL, Moser AH, et al. Role for circulating lipoproteins in protection from endotoxin toxicity. Infect Immun. 1995;63 (5):2041-2046. doi:10.1128/iai.63.5.2041-2046.1995

27. Bosmann M, Ward PA. The inflammatory response in sepsis. Trends Immunol. 2013;34(3):129-136. doi:10.1016/j.it.2012.09.004

28. Singer M, Deutschman CS, Seymour CW, et al. The third international consensus definitions for sepsis and septic shock (Sepsis-3). JAMA. 2016;315(8):801-810. doi:10.1001/jama.2016.0287

29. Fraunberger P, Schaefer S, Werdan K, et al. Reduction of circulating cholesterol and apolipoprotein levels during sepsis. Clin Chem Lab Med. 1999;37(3):357-362. doi:10.1515/CCLM.1999.059 
30. Golucci A, Marson FAL, Ribeiro AF, et al. Lipid profile associated with the systemic inflammatory response syndrome and sepsis in critically ill patients. Nutrition. 2018;55-56:7-14. doi:10.1016/j. nut.2018.04.007
31. Netea MG, Demacker PN, Kullberg BJ, et al. Low-density lipoprotein receptor-deficient mice are protected against lethal endotoxemia and severe gram-negative infections. J Clin Invest. 1996;97 (6):1366-1372. doi:10.1172/JCI118556

\section{Publish your work in this journal}

The International Journal of General Medicine is an international, peer-reviewed open-access journal that focuses on general and internal medicine, pathogenesis, epidemiology, diagnosis, monitoring and treatment protocols. The journal is characterized by the rapid reporting of reviews, original research and clinical studies across all disease areas. The manuscript management system is completely online and includes a very quick and fair peer-review system, which is all easy to use. Visit http://www.dovepress.com/ testimonials.php to read real quotes from published authors.

Submit your manuscript here: https://www.dovepress.com/international-journal-of-general-medicine-journal 